\title{
CMR-FD: Clustered Multipath Routing with False Destination to increase the Lifetime of Sensor Network
}

\author{
S. Manju Priya \\ Research Scholar Karpagam University \\ Coimbatore, Tamilnadu
}

\author{
S. Karthikeyan, PhD. \\ Asst.Professor in IT College of Applied Sciences \\ Sohar, Sultanate of Oman
}

\begin{abstract}
Routing in wireless sensor network is an important task. This has led to number of routing protocol which utilizes the limited resources. Since wireless sensors are powered by batteries, it is very essential to utilize their energy. Under these constraints many methods for conserving the power have been proposed to increase battery life. In this paper we propose a novel way by using clustered multipath routing with false destination (CMR-FD) to increase the lifetime of sensor nodes. It uses multiple paths between source and the destination which is intended to provide a consistent transmission with low energy.
\end{abstract}

\section{Keywords}

Sensor nodes, energy, lifetime, multipath

\section{INTRODUCTION}

Recent advances in micro electro mechanical systems, technology and wireless communications have led to small and low cost sensor network. The sensor network may comprise many application areas such as health monitoring, environmental monitoring including temperature, humidity, lightning condition, pressure etc. [23]. Additionally, many domain applications such as factory automation, chemical pollution monitoring, oil and gas remote monitoring, building sensor and security adopt sensor computing.

A mobile wireless sensor network consists of tiny sensor nodes which has three basic components: a sensing subsystem for data acquisition from the physical surrounding environment, a subsystem for local data processing and storing a wireless transmission subsystem for data transmission.

Furthermore, a battery is critical for a sensor node. All the sensors send data that they have sensed from the given region to the base station. The very important issue in this action is energy efficiency. There are other factors related to energy efficiency directly or indirectly like having a good routing protocol.

The path selected by nodes to send data is very affective because energy resources are limited and routing protocol should pay attention to this fact and has to send data in the path which doesn't lead the nodes to loss much energy.

Clustering is a technique used to reduce energy consumption of network nodes. The main contribution of this paper is using a multipath routing to send the data from one cluster to other clusters.

The remaining of this paper is organized as follow: section 2 guides an idea on prior works done in this area. Section 3 gives details about cluster formation and the proposed multipath routing, section 4 presents simulation results and performance analysis and section 5 presents conclusions and future work.

\section{RELATED WORK}

Wireless sensor networks have attracted much research in recent years. In order to minimize the energy consumption in WSN's several energy efficient routing protocols and algorithms has been developed $[1,2]$. Several researches have focused to provide energy efficiency routing protocols for wireless sensor network.

Another major issue in sensor networking is routing. The majority of the routing protocols can be classified into data centric, hierarchical, location based, network flow. Energy sensor node is assumed to know its own position as well as that of its neighbors which can be obtained with some localization schemes [7] [8]. Each node can forward packets to its neighbours within its transmission range that are closer to the sink node than itself.

A routing protocol for sensor networks should have scalability, data aggregation, network dynamics, low complexity, energy efficiency, fault tolerance and multiple paths. Multipath routing in ad hoc networks has been proposed in [17], [18], [19], [20]. For monitoring burst events a multipath routing protocol based on dynamic clustering and ant colony optimization is proposed in [13].

Partitioning the whole network into smaller areas can turn the network into an easily controllable and manageable infrastructure, and such grouping of sensors is the clustering. The main purpose of clustering is to use the network resources more efficiently, reduce overheads and provide a scalable architecture.

The cluster head plays its major role which mange's all other node belonging to its group. Due to this it may quickly cause energy fatigue. There has been some published work related to the cluster formation and the selection of cluster head [9] [10].

Generally, the clustering methods can be categorized into static and dynamic clustering. The static clustering aims at minimizing the total energy spent during the formation of the clusters for a set of network [11]. The dynamic clustering also deals with the same energy efficiency problem as in [12].

Some non uniform sensor deployment strategies have been proposed. Lian etal [3] focused on increasing the total data capacity by only considering the energy spent on the data transmissions. Reducing energy consumptions due to wasteful sources has been primarily addressed in the content of adaptive MAC protocols such as PAMAS (Power Aware Multi-Access Protocol) [4] and S-MAC [5, 6] 
In [22], by using trust mechanisms in MRBCH (Multi-Path Routing Protocol Based on Credible Cluster Heads) it creates the credit value, and based on the credit value the multi-path cluster head routing can finally be found. The credit value is created and exchanged among the cluster heads only. But this trust mechanism is used only for limited number of nodes.

In [14] the author uses a light weight minimum hop routing to send the packets from cluster head to another. Unequal clustering model [15] has focused on heterogeneous network where cluster head are deployed at some pre-computed locations.

In [21], the author advocates the use of a maximal leaf spanning tree for minimizing the number of forwarding nodes as part of an energy efficient broadcast mechanism in wireless sensor networks. However, it attempts only to maximize the number of leaf nodes without paying attention to energy level of intermediate nodes.

The authors B.Chen, K.Jameson, H.Balakrishnan, R.Morris in article [16], proposes an algorithm to turn off the nodes based on the necessity for neighbor's connectivity. It intends to reduce the system energy consumption without significantly diminishing the connectivity of the network.

\section{PROPOSED WORK}

Most of the protocols used in the routing find the minimum energy path or the shortest path routing. Always using these paths will soon make those nodes to lose their lifetime.

Multipath routing will distribute the traffic among multiple paths instead of routing through a single path. The proposed plan is to first find out the neighbour node list and then to find the multiple path from the neighbour nodes. The data packets are distributed through the multiple paths to the destination. As in the previous paper [23], the route is identified, but if any leaf node is found, further process is done. The work is been divided into 5 stages:
(i) Initialization stage
(ii) Finding neighbour nodes
(iii) Finding multipath
(iv) Data transmission
(v) Maintenance stage

\subsection{Initialization}

In this stage, the nodes are first clustered and the "HELLO" packet message is send to all the nodes. The node which has more energy is elected as cluster head $(\mathrm{CH})$. The remaining nodes are treated as member nodes of that cluster. The node which has the next energy level to the cluster head is treated as next_CH. In situation like when the cluster head loses its energy level below the threshold value, then the next_CH will act as the cluster head and the current head goes to sleep mode.

After sending the data, the cluster head may lose some energy. The remaining energy of the cluster head is calculated by the formula.

Remaining energy $=$ I.E. $-(($ No. of packets transmitted $*$ T.E. $)+($ No. of packets received * E.C. $))$

Where

I.E. - initial energy of the node
T.E - transmission energy to transmit the packet

E.C. - energy consumed by receiving a packet

If the remaining energy < energy of NEXT_CH, then a MSG from the current head is passed to the NEXT_CH to act as the cluster head for the next iteration. If the remaining energy > energy of NEXT_CH, then there will be no change in the election of cluster head.

\subsection{Find neighbor nodes}

Before finding the multipath, create a neighbour table for the source nodes. The steps are as follows:

max_node -number of nodes in the network

$\mathrm{x}, \mathrm{y} \quad-$ positions of the current node

node_id -node number

th - threshold energy

R- Residual energy of the node

G_node - nodes within the transmission range of current node

Trans_range - transmission range

1. Get the value of maximum nodes in each cluster

2. Get the position of source node

3. Let the source node be with node id $=0$

4. Find the distance between the source node and all other nodes using the distance formula

$$
\operatorname{dis}=\sqrt{ }\left(\mathrm{y}_{2}-\mathrm{y}_{1}\right)^{2}+\left(\mathrm{x}_{2}-\mathrm{x}_{1}\right)^{2}
$$

where $\left(\mathrm{x}_{1}, \mathrm{y}_{1}\right)$ are the positions of the source node and $\left(\mathrm{x}_{2}, \mathrm{y}_{2}\right)$ are the positions of the node from which the distance is to be calculate.

5. if (dis<trans_range) then update the neighbours of the source node in the neighbour list

In case if the distance is within the transmission range and the node energy is greater than the threshold energy then update the neighbour list of the source node. Consider the following fig. 1 , node 1 is acting as the source and node 11 is the destination. 


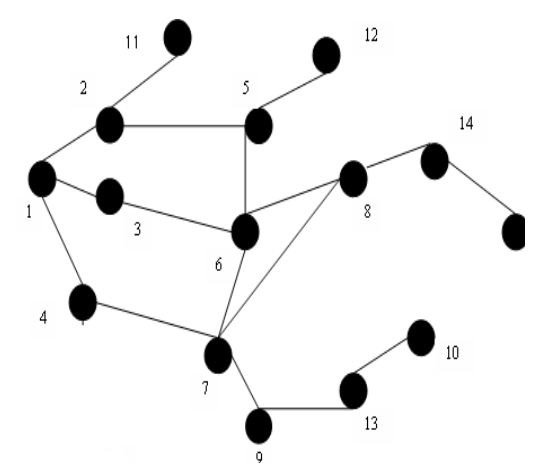

Fig.1: Network model

For the above figure, the neighbour list is as follows:

Table 1: Neighbour list

\begin{tabular}{|l|l|}
\hline Node & Neighbour list \\
\hline 1 & $2,3,4$ \\
\hline 2 & $1,5,11$ \\
\hline 3 & 1,6 \\
\hline 4 & 1,7 \\
\hline 5 & $2,6,12$ \\
\hline 6 & $3,5,7,8$ \\
\hline 7 & $4,6,8,9$ \\
\hline 8 & $6,7,14$ \\
\hline 9 & 7,13 \\
\hline 10 & 13 \\
\hline 11 & 2 \\
\hline 12 & 5 \\
\hline 13 & 9,10 \\
\hline 14 & 8,15 \\
\hline 15 & 14 \\
\hline
\end{tabular}

From the neighbour_list() eliminate the leaf node if it is not source or destination. The leaf nodes are those connected by one edge. Leaf node removal can result in new leaf nodes, acyclic graph, isolated node or an empty graph. A set of removed leaf nodes is referred as ordered nodes. The neighbour node list is ordered until no further elimination of leaf node is possible.

\section{Algorithm for removing leaf node from neighbour_list ()}

Step 1: Initially all the nodes are in wake state

Check in neighbour_list()

While leaf node exists do

if leaf node $==$ source node

keep the source node in wake state itself

else

\author{
if leaf node $==$ destination node) \\ assign false destination <- leaf node \\ else \\ Sleep<- leaf node \\ Endif \\ End if
}

Step 2: The while loop is repeated until no further elimination is possible.

Step 3: After removing the leaf node, the new list_neighbour(s) is formed.

Step 4: All the leaf nodes are set to sleep mode.

\subsection{Finding Multipath}

The next step is to find the available path for the source to reach the destination in such a way that no node is repeated in the path.

Now the neighbours for the source node 4 are 7 and 1 . Through nodes 7 and 1 the data can be passed. The path is found out in such a way that no node is repeated again in the path. In case if the destination node is the leaf node, use a single path between false destination to reach destination. Similarly no node is repeated in the path because if any two different paths come across to same node, there can occur collision or traffic in the network. It may lose its energy or data. So in the algorithm the repeated node is avoided.

\section{Algorithm to find the route:}

Consider the below parameters:

Start_node - source node

Finish_node - destination node

Intermediate_node - the next node added in the path

Path - list of nodes on the path including start_node and finish_node

Visited_node - list of nodes already visited

False_destination - if the destination node is the leaf node

Step 1: Get the nodes from the list_neighbour(s)

Step 2: For each neighbour_node, find the next intermediate node. If intermediate node of first path is present in second path, avoid that node in the path and move to next path.

Step 3: Repeat the step 2 until it reaches the destination

Step 4: if the destination is false_destination, then the data is passed from the source to false destination through multiple paths. From the false_destination, the data is send to the destination_node through single path.

Step 5: Else, the multiple path is followed.

The available path for the above network is

Path1: 4-> 7->6->5->2

Path2: 4-> 1->2

Path3: 4->7->6->3->1->2

Path4: 4->1->3->6->5->2 
Here node 2 is acted as false destination. By using the algorithm we can find that in path 4 , the nodes 6 and 5 are repeated. So path 4 is avoided. Similarly the path 3 is also eliminated since node 6 and node 7 are repeated.

So the available path is

Path1: 4->7->6->5->2

Path2: 4-> 1->2

After finding the path a RREQ message is send to the false destination from the source. Once when the RREP message reached to the source, it sends the data to the false destination through the available paths.

From source node 4 to node 2 , multiple path routing is followed. From the false _destination (node 2) the data is send to the destination node (node 11) through single path.(as shown in fig. 2)

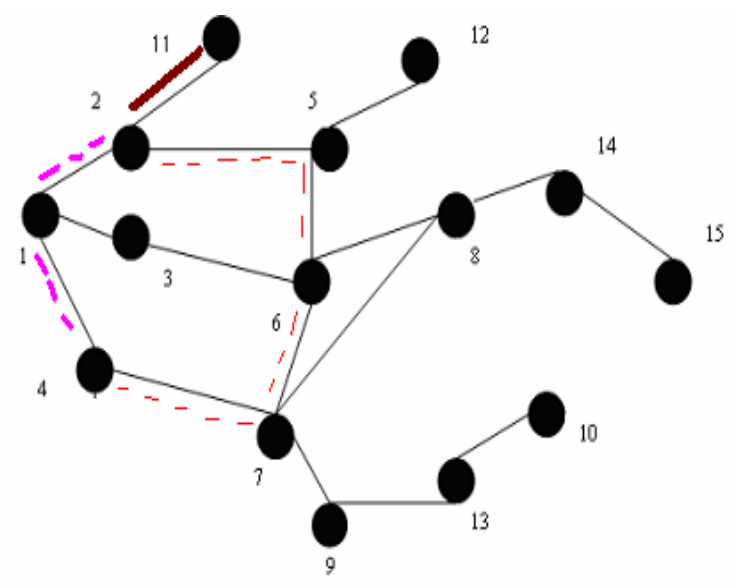

Fig2. Path from node 4 to node 11

In all these paths no node is repeated or duplicated. Since the data packets are send through 2 different paths there is no chance for traffic or collision in the network. In this multipath routing, it has used only 5 nodes to send the packets (nearly $40 \%$ of nodes) and all other nodes are not used. By turning off the leaf nodes it saves energy.

\subsection{Data Transmission}

This clustered multipath algorithm using false destination (CMR-FD) can be used for both inter as well as intra clustering. First, in intra clustering, the cluster head $(\mathrm{CH})$ distributed the time division multiple address (TDMA) time slots for all member nodes of the cluster. Each member node sends data to the $\mathrm{CH}$ in its own time slot, for other time it can be in sleep mode to save energy.

Second, the inter clustering of data. Since the proposed algorithm constitutes a connected graph by using its neighbours between the cluster head and base station, the data transmission is quite different from other clustering routing protocols. The cluster head has the responsibility of collecting all the data from the member node and send it to the base station. The multiple path is used to send the data from one $\mathrm{CH}$ to another $\mathrm{CH}$ to reach the destination.

Based on the application, each $\mathrm{CH}$ send to BS based on the tuples X (Neighbour_list, E_nodes, E_BS, time_delay) where neighbour_list - list of neighbours

E_nodes - energy of each node

E_BS - energy of the base station

time-delay - minimum time delay through $\mathrm{CH}$ to $\mathrm{BS}$

First we consider that all $\mathrm{CH}$ transmits data to the BS through their path, so the inter-cluster energy consumption of data transmission is minimum. Secondly, we consider the residual energy ( $\mathrm{E}$ node) and the minimum energy consumption to reach the base station (E_BS). When each $\mathrm{CH}$ transmits the data, the energy is calculated as

Trans_Eenrgy(i) $=z^{*}\left(E(\mathrm{i}) / \mathrm{E} \_a v g E\right)+(1-\mathrm{z})(\mathrm{Th} / \mathrm{E}-\mathrm{BS})$ where

$\mathrm{z}$ - weighting coefficient which dynamically balances the node residual energy and minimum energy consumption to BS

$\mathrm{E}(\mathrm{i})$ - current residual energy of $\mathrm{CH}$

E_avgE - average energy of all other nodes in the path

Th- threshold energy

E-BS - minimum energy from $\mathrm{Ch}$ to BS

From the above discussion, it is found that there is more advantage whole using the CMR-FD to transmit the data. It improves the reliability, flexibility of the routing. Based on E_nodes we can balance the E_BS, minimize the energy consumption of inter-cluster data transmission and can save energy.

\subsection{Maintenance}

When sending the data from the source to destination, there can occur any breakage in the route. The node which discovers the link breakage between two nodes, it sends a route error (RERR) message to the backward direction to the source node. From the neighbour list table, the source node uses an alternate valid route.

\section{SIMULATION RESULTS AND PERFORMANCE ANALYSIS}

\subsection{Simulation Results}

This section presents and discusses the simulation results and performance study of CMR-FD. The proposed sensor network consists of $\mathrm{N}$ nodes ( $\mathrm{N}$ varies from 50 to 300 nodes) nodes that randomly dispersed in $100 \times 100$ of square fields with a single base station. All parameters of simulations are shown in Table 2. All nodes are identical with a radio transmission range set to $25 \mathrm{~m}$. To check the energy consumption of the algorithm the energy level of each node is considered $300 \mathrm{~mJ}$. 
Table 2. Simulation Parameters

\begin{tabular}{|l|l|}
\hline \multicolumn{1}{|c|}{ Parameter } & \multicolumn{1}{c|}{ Value } \\
\hline Network Filed & $(0,0) \sim(100,100)$ \\
\hline Node numbers & $50 \sim 300$ \\
\hline Cluster radius $r$ & $50 \mathrm{~m}$ \\
\hline Sensing radius $r s$ & $15 \mathrm{~m}$ \\
\hline Sink position & $(50,200)$ \\
\hline Data packet size & 256 Bytes \\
\hline Broadcast packet size & 10 Byte \\
\hline Ethreshold & $0.01 \mathrm{~J}$ \\
\hline Transmit Power & $1.2 \mathrm{w}$ \\
\hline Receiving Power & $1.2 \mathrm{w}$ \\
\hline Idle Power & 12 Units \\
\hline Sleep mode Power & 0.015 Unit \\
\hline
\end{tabular}

The figure below (fig.3) shows the network routing for 100 nodes with different routing path from all the nodes.

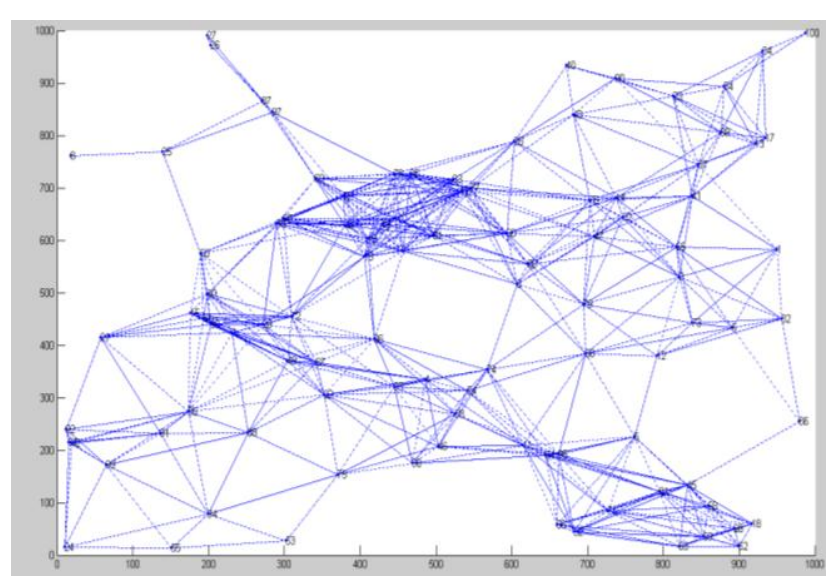

Fig.3: Routing from all nodes

\subsection{Comparison Results}

\section{(i) Packet delivery ratio:}

Packet delivery ration for the proposed CMR-FD increases when compared to LEACH, MRBCH. (as shown in fig.4). The utilization of immediate selection of routing path incase of any error, improves the packet delivery ratio.

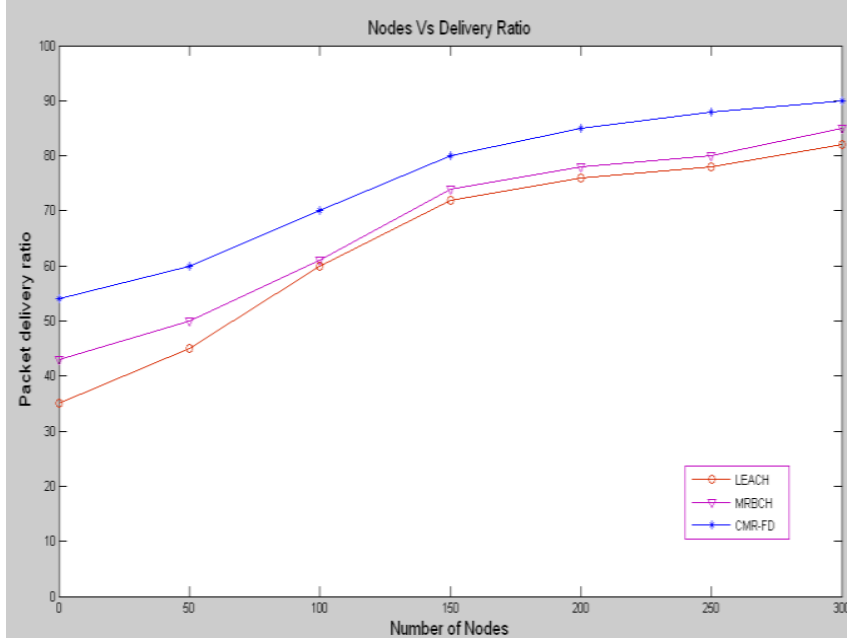

Fig.4: Packet delivery ratio

\section{(ii) Energy Consumption}

Under energy constraints, it is vital for sensor nodes to minimize energy consumption in radio communication to extend the lifetime of sensor networks. From the results, as shown in the fig. 5, it is observed that the proposed method consumes less energy than other methods. When the node density increases $\mathrm{MRBCH}$ has higher energy consumption and at the mean time, the it used fixed amount of energy, since only limited numbers of nodes are utilized while the other nodes are in sleep mode.

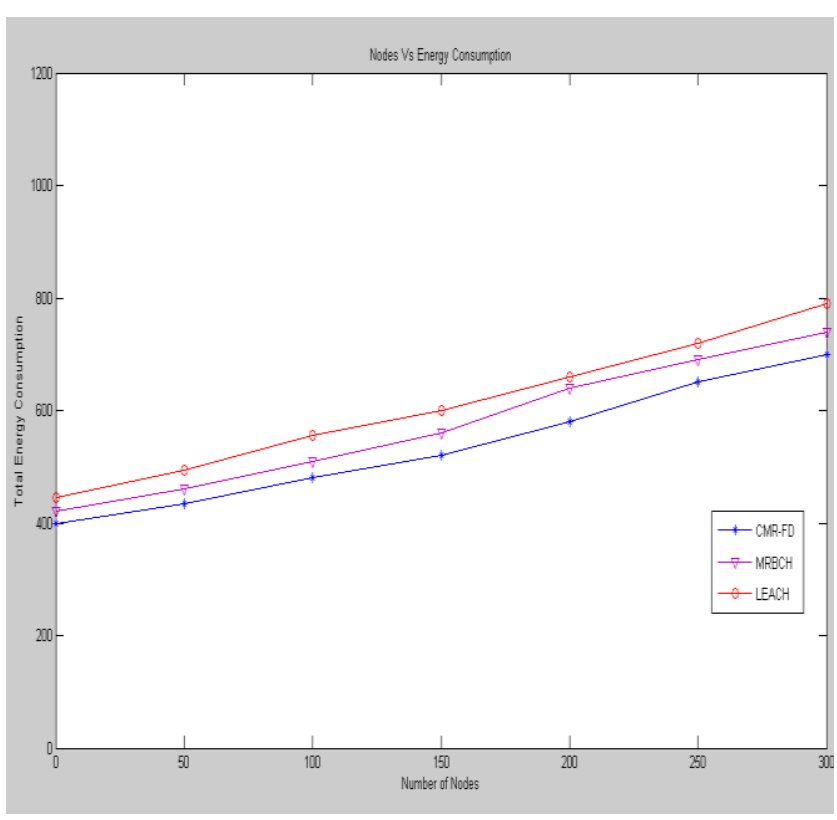

Fig.5: Energy Consumption

(iii) Average-end-end delay:

It is the averaged over all surviving data packets from the source to the destination. From the fig. 6, it proofs that the proposed routing is less when compared to other routing. Moreover this result shows that energy efficient cluster head multipath routing with FD has the ability to sustain more performance for larger nodes. 


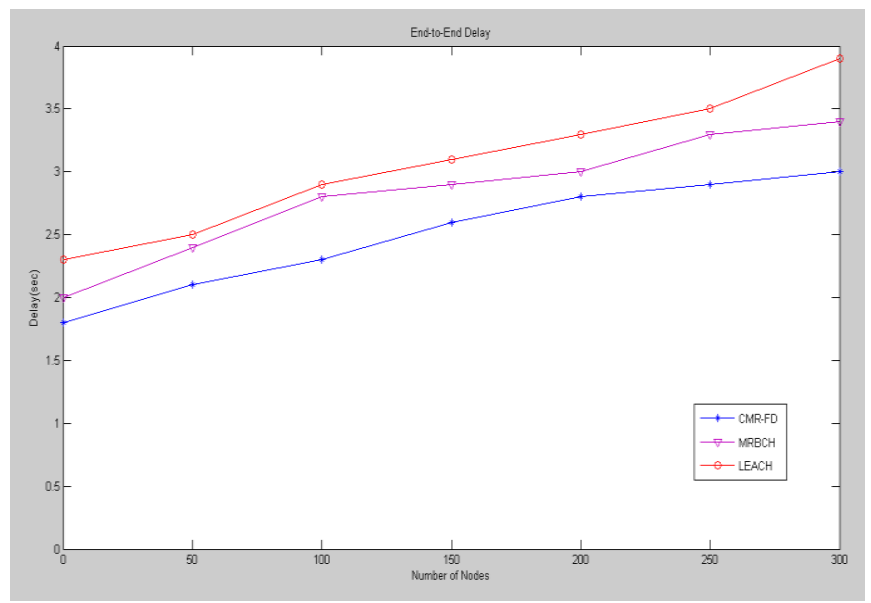

Fig.6: Average-end-end-delay

\section{CONCLUSION}

Based on the energy of the cluster head, we propose the CMR-FD, for sensor networks. It has the advantages like prolong lifetime of the whole network by using multi-path route for data transfer, making use of the multi-path clusterhead routing based on the energy of the nodes, and making the leaf node to sleep state, save more energy. Simulation results and comparisons shows that this protocol can save the network resources, increase the network lifetime. Our future work will be further investigating in multipath clustering techniques for real time applications.

\section{REFERENCES}

[1] A.Boukerche, I.Chatzigiannankis and S.Nikoletseas, Computer Communication, Vol 4, No.29, Feb( 2008), pp.477A New Energy Efficient and Fault Tolerant Protocol for Data Propagation in smart dust Networks using varying transmission range

[2] J.Gao and L.Zhang , (2004), pp. 1099-1108, in Proc "Load Balanced short Path Routing in Wireless Networks", Proc, IEE INFOCOM '04

[3] J.Lian, K.Naik and G.B.Agnew, Vol2. No.2, (2006), pp.121-145. Data Capacity Improvement of wireless sensor networks using non-Uniform sensor distribution", Int'l Distributed Sensor Networks

[4] S.Singh and C.Raghavendra, ACM Computer Communciation Vol.28 No.3, July (1998), pp5-26 ,PAMAS:Power Aware MultiAccess protocol with signaling for Ad-hoc Networks

[5] W.Ye.J.Heidenmann and D.Esteing, June (2002),An Energy Efficienct MAC Protocol for wireless sensor network in Proceedings of IEEE INFOCOM, NewYork,

[6] Di Tian and Nicolas D. Georganas, (2002), pp.32-41.A Coverage-Preserving Node Scheduling Scheme for Large Wireless Sensor Networks", iSENSE Project, Communications and Information Technology Ontario (CITO),

[7]. L.Doherty, L.El Ghaoni and K.S.J Pister, in Proc, IEEE INFOCOM April (2001), pp.1655-1663, Convex position estimation in wireless sensor networks

[8]. Y.Shang, W.Ruml, Y.Zhang and M.P.J.Fromherz, in Proc MobilComm, June (2003), pp.201-212 "Localization from mere connectivity"
[9] W.R.Heinzelman, A.P. Chandrakasan, H.Balakrishnan, "An Application specific protocol architecture for wireless micro sensor networks" IEEE Transactions on Wireless communication, pp.660-670, (2004).

[10] M.Kochhal, L.Schwiebert, S.Gupta, "Role base hierarchical self organization for wireless ad-hoc sensor networks" in Proc, of second ACM International Workshop on wireless sensor networks and applications (WSNA “03” ACM Press,(2003).

[11] S.Bandyopadhyay and E.J.Coyle, "Minimizing communication Costs in hierarchically clustered networks of wireless sensors", Computer Networks, Vol. 44, no.1, pp.1-16, (2004).

[12] Y.Ma and J.H.Aylor, "System Lifetime Optimization for heterogeneous sensor networks with a hub-spoke topology", IEEE Trans. Mobile computing, Vol.3, no.3, pp.286-294, (2004).

[13] Jing Yang 1,2,*, Mai Xu 3,4, Wei Zhao 4 and Baoguo Xu." A Multipath Routing Protocol Based on Clustering and Ant Colony Optimization for Wireless Sensor Networks “Article, Sensors (2010), 10, 4521- 4540

[14] S.Sero and W.Heinzleman, "Prolonging the Lifetime of wireless sensor network's via unequal clustering" Proc IEEE International Parallel and distributed Processing Symposium, NewYork, (2005).

[15] Prakash G L, Chaitra K Meti, Poojitha K, Divya R.K., "Effect of Clustering on Energy Efficiency and Network Lifetime in Wireless Sensor Networks", World Academy of Science, Engineering and Technology , (2009)

[16] B.Chen, K.Jameson, H.Balakrishnan, R.Morris, "Span: An Energy Efficient Coordiantion Algorithm for Topology Maintennance in Ad-hoc Wireless Network", (2001).

[17] S.-J. Lee and M. Gerla, "AODV-BR: Backup Routing in Ad hoc Networks,"

Proceedings of IEEE WCNC 2000, Chicago, IL, Sep. (2000).

[18] A. Nasipuri and S.R. Das, "On-Demand Multipath Routing forMobile Ad Hoc Networks," Proceedings of IEEE ICCCN'99, Boston, MA, Oct. (1999), pp. 64-70.

[19] V.D. Park and M.S. Corson, "A Highly Adaptive Distributed Routing Algorithm for Mobile Wireless Networks," Proceedings of IEEE INFOCOM' 97, Kobe, Japan, Apr.(1997), pp. 1405-1413.

[20] J. Raju and J.J. Garcia-Luna-Aceves, "A New Approach to On-demand Loop-Free Multipath Routing," Proceedings of IEEE ICCCN'99, Boston, MA, Oct. (1999), pp. 522-527.

[21]. B.Zeng, L.Yao and Y.Q.He, “An Energy Efficient Broadcast Control Protocol for Wireless Sensor Networks", Proceedings of the IEEE International Conference on Networking , Architecture and Storage, pp. 3-8, (2009).

[22]. Yang Yang, Enjian Bai, Jia Hu, Wenqiang Wu ,' MRBCH: A Multi-Path Routing Protocol Based on Credible Cluster Heads for Wireless Sensor Networks" Int. J. Communications, Network and System Sciences, 3, 689-696, (2010)

[23]. S.Manju Priya, Dr. S. Karthikeyan, 2012, IJCSI International Journal of Computer Science Issues An Efficient Clustered Multipath Routing to improve lifespan in WSN, Vol. 9, Issue 2, No 2. 